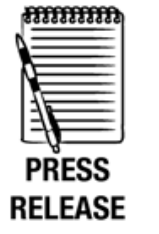

- Additional materials are published online only. To view these files please visit the journal online (http://oem.bmj.com).

${ }^{1}$ Centre for Research in Environmental Epidemiology (CREAL), Hospital del Mar Research Institute (IMIM), CIBER Epidemiologia y Salud Pública (CIBERESP), Barcelona, Spain

${ }^{2}$ Formerly (except MM and ID) at International Agency for Research on Cancer, Lyon, France

${ }^{3}$ France Telecom RD, Issy-les-Moulineaux, France ${ }^{4}$ Engineering and Physical Hazards Branch, National Institute for Occupational Safety and Health (NIOSH), Cincinnati, Ohio, USA

${ }^{5}$ Institute of Cancer Epidemiology, Danish Cancer Society, Copenhagen, Denmark ${ }^{6}$ Centre for Radiation, Chemical and Environmental Hazards, Health Protection Agency,

Didcot, UK

${ }^{7}$ Department of Electrical and Electronic Engineering, Tokyo Metropolitan University, Tokyo, Japan

${ }^{8}$ EMC Group, Applied Electromagnetic Research Center, National Institute of Information and

Communications Technology, Hachioji, Tokyo, Japan ${ }^{9}$ Department Technology and Health, National Institute of Health, Rome, Italy

\section{Correspondence to}

Professor E Cardis, Centre for Research in Environmental Epidemiology (CREAL), Hospital del Mar Research Institute (IMIM), CIBER Epidemiologia y Salud PÃblica (CIBERESP),

Doctor Aiguader 88, 08003

Barcelona, Spain;

ecardis@creal.cat

Accepted 20 May 2011 Published Online First 9 June 2011

This paper is freely available online under the BMJ Journals unlocked scheme, see http:// oem.bmi.com/site/about/ unlocked.xhtml

\title{
Estimation of RF energy absorbed in the brain from mobile phones in the Interphone Study
}

\author{
E Cardis, ${ }^{1,2}$ N Varsier, ${ }^{3}$ J D Bowman, ${ }^{4}$ I Deltour, ${ }^{5,2}$ J Figuerola, ${ }^{1}$ S Mann, ${ }^{6}$ \\ M Moissonnier, ${ }^{2} \mathrm{M}$ Taki, ${ }^{7,8} \mathrm{P}$ Vecchia, ${ }^{9} \mathrm{R}$ Villegas, ${ }^{1} \mathrm{M}$ Vrijheid, ${ }^{1,2} \mathrm{~K} \mathrm{Wake}^{9}{ }^{9} \mathrm{~J}$ Wiart $^{3}$
}

\section{ABSTRACT}

Objectives The objective of this study was to develop an estimate of a radio frequency (RF) dose as the amount of mobile phone RF energy absorbed at the location of a brain tumour, for use in the Interphone Epidemiological Study.

Methods We systematically evaluated and quantified all the main parameters thought to influence the amount of specific RF energy absorbed in the brain from mobile telephone use. For this, we identified the likely important determinants of RF specific energy absorption rate during protocol and questionnaire design, we collected information from study subjects, network operators and laboratories involved in specific energy absorption rate measurements and we studied potential modifiers of phone output through the use of software-modified phones. Data collected were analysed to assess the relative importance of the different factors, leading to the development of an algorithm to evaluate the total cumulative specific RF energy (in joules per kilogram), or dose, absorbed at a particular location in the brain. This algorithm was applied to Interphone Study subjects in five countries.

Results The main determinants of total cumulative specific RF energy from mobile phones were communication system and frequency band, location in the brain and amount and duration of mobile phone use. Though there was substantial agreement between categorisation of subjects by cumulative specific RF energy and cumulative call time, misclassification was non-negligible, particularly at higher frequency bands. Factors such as adaptive power control (except in Code Division Multiple Access networks), discontinuous transmission and conditions of phone use were found to have a relatively minor influence on total cumulative specific RF energy.

Conclusions While amount and duration of use are important determinants of RF dose in the brain, their impact can be substantially modified by communication system, frequency band and location in the brain. It is important to take these into account in analyses of risk of brain tumours from RF exposure from mobile phones.

\section{INTRODUCTION}

The multinational epidemiological study Interphone $e^{1}$ was conducted to evaluate the possible relationship between risk of brain, acoustic nerve and parotid gland tumours and radio frequency (RF) exposure from mobile phones. Most epidemiological papers to date have evaluated risk only in relation to reported mobile phone use. ${ }^{2-21}$

The relation between mobile phone use and RF dose (expressed as energy absorption) at the

\section{What this paper adds}

- Epidemiological studies of brain tumours in relation to mobile phone use have mainly analysed risk in relation to amount and duration of use of mobile phones.

- While these factors are important determinants of the amount of radio frequency energy in the brain, our results suggest that their impact can be substantially modified by communication system, frequency band and tumour location.

- It is therefore important to take these into account in analyses of risk of brain tumours in relation to radio frequency exposure in Interphone and future case-control and cohort studies.

location of the tumour is complex, however, particularly since dose is localised. The Japanese Interphone Study ${ }^{20}$ developed dose indices taking localised absorption into account by multiplying phone duration by spatial maximum specific energy absorption rate (SAR) in the tumour region. SAR, in watts per kilogram, represents absorbed power per unit mass of tissue, a widely used metric of RF dose rate. ${ }^{22} 23$

Many parameters can, however, influence amount and distribution of RF dose in the brain, ${ }^{20} 2425$ including telephone type, network characteristics, and amount and conditions of phone use.

This paper presents an algorithm, developed within Interphone, to account for these parameters for dose estimation in epidemiological studies and illustrates its application on study subjects from five Interphone countries (Australia, Canada, France, Israel and New Zealand).

\section{METHODS}

Interphone is a collaborative case-control study conducted in 13 countries (Australia, Canada, Denmark, Finland, France, Germany, Israel, Italy, Japan, New Zealand, Norway, Sweden and the UK). Study methods and results of analyses of brain tumour risk related to mobile phone use have been published. ${ }^{1} 61112$ 14-16 18-21

Subjects were interviewed using a questionnaire, including a mobile phone use calendar, structured according to factors that could change patterns of phone use and output power. Hundreds of pictures of mobile phones were compiled to assist subjects 
in identifying models used. Phone use for each subject was reported by period of use for each phone; if use changed, multiple periods were reported. For each period, questions were completed concerning network operator and duration and number of calls. Information on laterality, call environment and use of hands-free devices was collected. Information was also collected about Digital Enhanced Cordless Telecommunications (DECT) telephones. As use was limited among study subjects and because both peak and mean output are lower for mobile phones in the study, they were not included in the RF dose estimation.

An important goal was to collect all information necessary for estimation of the subject's RF dose (figure 1):

- Responses of individual subjects to the questionnaire (above);

- Diagnostic images and report for cases, which permitted neuroradiologists to localise the tumour and its estimated centre on a 3-dimensional (3D) grid, the Gridmaster, ${ }^{26}$ so that RF dose at that location could be evaluated;

- Historical information on communication technologies and network characteristics obtained by questionnaire from network operators and from published documents and standards. The questionnaire concerned history of different communication systems and frequency bands, enablement of adaptive power control (APC), discontinuous transmission (DTX) and percentage of traffic in each band or system (where multiple bands/systems were in operation);

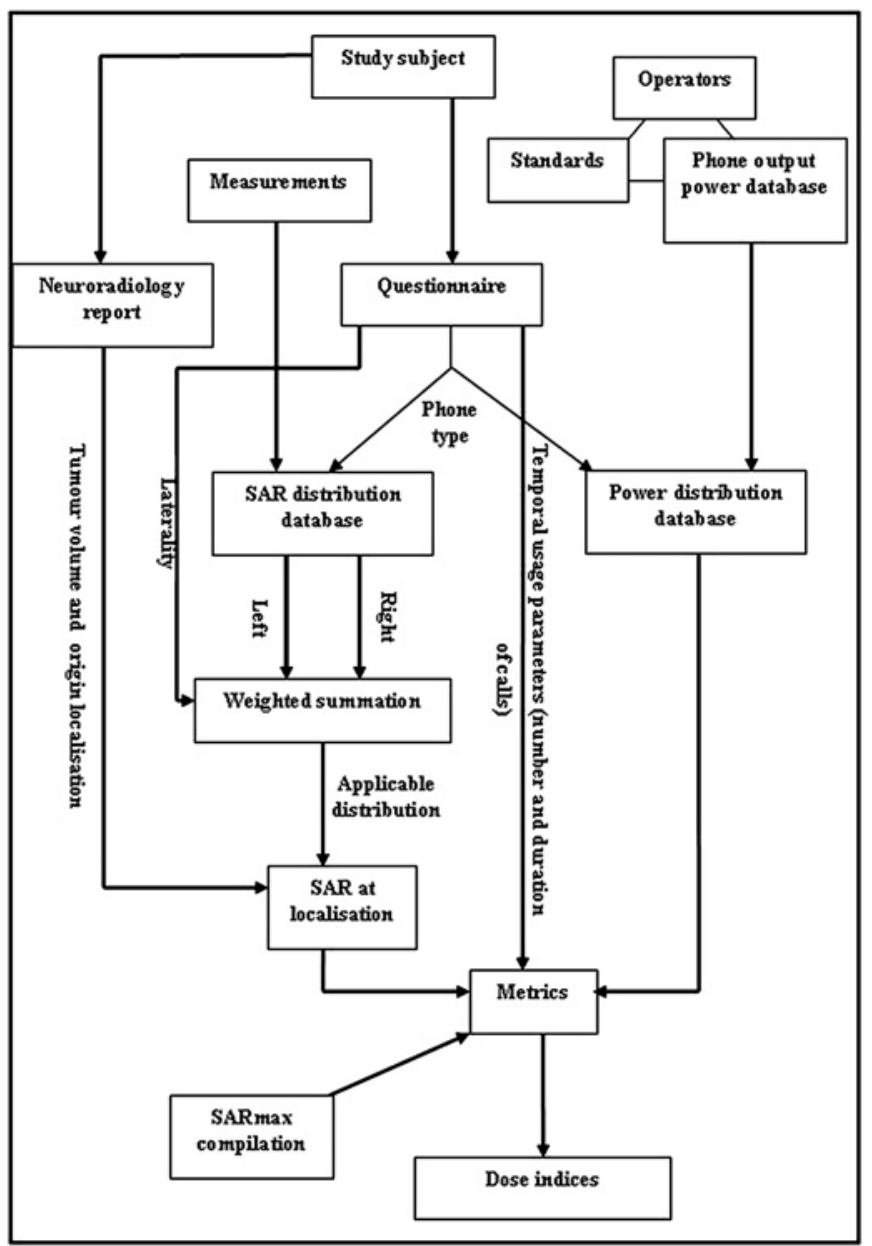

Figure 1 Combining the information collected in the Interphone study into an RF dose estimate
- A database of SAR spatial distributions in the head, based on phantom dosimetric measurements made in France Telecom $\mathrm{RD}$ laboratory and Japanese Telecom Engineering Center ${ }^{27}$;

- A compilation of published SARmax values (SAR measured with phone emitting at maximum power), averaged over 1 or $10 \mathrm{~g}$ of tissue, depending on applicable standards, for a large number of phones commercialised during and before the study;

- A phone output power database, derived from the use in most Interphone countries of software-modified Global System for Mobile (GSM) phones (SMPs), which stored usage, power and frequency data. ${ }^{28}$ Some measurements were also made in non-GSM networks.

\section{Mobile communication systems}

Mobile telephone use started in most Interphone countries between the mid- and late 1980's. Communication systems are often categorised into generations, with early analogue systems classified as first generation, early digital systems as second generation and recent digital systems with enhanced data capability as third generation. Characteristics of communication systems, including analogue/digital technology, frequency band, emitted power and enablement of APC, are elements that may affect RF dose from mobile phones. The phone systems relevant for Interphone countries, together with their history and characteristics, are discussed in online appendix 1.

\section{Elements of the RF dose algorithm}

SAR distributions from mobile phones

Hundreds of different phone models were reported by Interphone Study subjects. It was essential to determine whether brain radiation patterns differ between phones and, if so, to develop a method for assessing SAR distribution from different models.

Computer simulations and experiments using phantoms (representing a head filled with homogeneous liquid) are the main dosimetric approaches used to produce an SAR distribution from a mobile phone, emitting in a specific frequency band, held in a specific position and radiating a specific power. Work involved is considerable, and it was not practical to conduct such detailed dosimetry for every phone model used.

Results of SAR dosimetric measurements made in two broadly used standard phantoms (Specific Anthropomorphic Mannequin ${ }^{22}$ and Generic Twin $^{29}{ }^{30}$ ) were therefore analysed to derive 3D SAR distributions in the brain for mobile phones used by the study subjects and assign absolute levels of dose in the most exposed brain area. The approach, shown in online appendix figure 1, is detailed below.

\section{Identification of phone classes}

The aim was to identify phone classes producing distinct spatial distributions in the brain into which phones used historically by study subjects could be classified. Analyses of SAR spatial distribution were conducted using dosimetric databases from France and Japan on over 100 phone models from different countries and time periods, ${ }^{27}$ and clusters of SAR distributions were sought in relation to external phone features and communication system. The method is described in detail elsewhere. ${ }^{31}$

\section{$3 D$ distribution of SAR in Gridmaster}

Each phone in the measurement database had finely sampled $(1 \mathrm{~mm})$ data only in the vicinity of the spatial peak SAR (close to the phone) and no measurement throughout large portions of the head (online appendix figure 2). A method was developed to estimate average SAR in each cube of the $1 \mathrm{~cm}^{3}$ Gridmaster so that SAR at the location of each tumour could be derived 
( $\mathrm{see}^{32}$ for details). For each cube in the vicinity of the spatial peak, the finely sampled SAR measurements were averaged. The parameter of the exponential decay function inside the brain was estimated and average SAR in $1 \mathrm{~cm}^{3}$ extrapolated to the entire Gridmaster. Validation of the method was performed experimentally and numerically. ${ }^{32}$

\section{D SAR distributions for each phone class}

The spatial SAR distribution in a phone class was obtained by deriving the average, median and range of SAR values in each cube from all phones in the class. The relative average SAR value was computed in each Gridmaster cube as the ratio of its average SAR to the average in the cube with the highest SAR (generally located in the outer layer of the grid near the ear). Detailed methods have been published. ${ }^{27}$

\section{Output power levels}

A mobile phone's RF emissions are directly proportional to its output power, which may vary substantially between different networks (depending on infrastructure at a given time, communication system, frequency band, enablement of different transmission protocols, such as DTX) and phone use circumstances (moving/stationary, urban/rural settings) due to power control technologies, such as APC. Determinants of output power were studied in volunteers in 12 countries who used GSM SMPs for approximately 1 month each. Detailed methods and results have been published. ${ }^{28}$ The SMPs recorded frequency band and output power at fixed sampling intervals throughout calls. Questionnaires provided information on typical circumstances of the volunteer's phone use. Regression models were used to analyse influence of possible explanatory variables on output power of the phones.

\section{Phone-specific SAR values}

The SARmax of phones varies considerably with phone model and was thought to possibly relate to marketing period. Reported SARmax values for particular phone models were compiled from all accessible sources, including manufacturers' websites, formal comparison studies and the 'grey' literature (including consumer magazines and websites). This dosimetry information was compiled into a database, including information on phone characteristics, measuring laboratory, phantoms and measurement protocols.

\section{Statistical analyses}

Descriptive analyses of the resulting dose estimate were conducted, producing standard summary statistics. Agreement between classification of subjects by categories of dose and of mobile phone history was evaluated using a weighted $\kappa$ statistic. ${ }^{33}$ Analysis of variance was conducted to evaluate the relative importance of different factors in dose estimation.

\section{RESULTS \\ SAR distributions from mobile phones} Identification of phone classes

Analysis showed some clustering of phone types related to the combination of phone position and shape. ${ }^{31}$ The position in which phones are held varies between individuals and, for the same individual, during use and with different phones. Classifications of SAR distributions using phone position therefore cannot be used in epidemiological studies.

Frequency is known to be inversely related to penetration depth of radiation. ${ }^{34}$ Phones were therefore classified by band as follows: 800-900, 1500 and 1800-1900 MHz. No measurement and limited simulations were available on $450 \mathrm{MHz}$ phones, and only 14 subjects in the five countries reported ever using them; they were therefore included with $800-900 \mathrm{MHz}$ phones.

\section{Average spatial SAR distribution for each phone class}

From each phone measured, 3D SAR distributions were estimated in the Gridmaster and, for each phone class, the average SAR distribution was derived. Figure 2 shows average SAR distributions in the brain at 800-900 and $1800 \mathrm{MHz}$.

Most of the SAR in the brain $(97 \%-99 \%$ depending on frequency band) appeared to be absorbed in the hemisphere on the side where the phone is used, mainly $(50 \%-60 \%)$ in the temporal lobe. The average relative SAR decreased rapidly with depth, particularly at higher frequencies (figure 2). The SAR distribution appeared similar across phone models, between older and newer phones, and between phones with different antenna types and positions. ${ }^{27}$

\section{Output power levels}

\section{Influence of APC}

In GSM phones, before APC was introduced in the early 1990s, phones worked to maximum power. The SMP study conducted between 2001 and 2005 with four GSM models, and over 63000 calls made by 516 subjects in 12 countries, showed that, on average, APC reduced power to around $50 \%$ of the maximum power levels in both 900 and $1800 \mathrm{MHz}$ frequency bands. ${ }^{28}$ Results from this and other studies ${ }^{35-40}$ are broadly consistent in the period 1999-2005 for GSM networks.

For any historical dose index, however, extrapolation in time is necessary. GSM networks were introduced in early to mid1990s in Europe and more recently in North America. Earlier, first-generation analogue networks had no or very limited APC capability (K Hansson Mild, personal communication, 2007), so phones were nearly always operating at maximum power. Since the start of GSM systems, improved network coverage may have reduced power levels, but increased indoor use of phones, more frequent use of handovers and higher traffic density may have increased them. Thus, there are uncertainties about backward extrapolation and we decided, for GSM and other secondgeneration networks, to assume a factor of 1 for APC up to its introduction and of 0.5 afterwards.

For Code Division Multiple Access (CDMA) networks, information about power control is scant, except that systems are very efficient. In a limited number of measurements in Canada, the average power was 13-15 times less than peak; an APC factor of 0.067 was therefore chosen for CDMA networks, compatible with recently published data comparing CDMA and GSM exposure levels. ${ }^{41}$

\section{Influence of network operator}

The SMP study indicated important differences between operators. $^{28}$ Within a frequency band, operator was the most important factor explaining differences in average power levels between users. The study, however, was only a snapshot in time and place, and network optimisation factors vary considerably over time and place. SMP volunteers moreover did not form a geographically representative sample of the study population in most countries, and no information was available for some networks in study regions. For these reasons, though this adds uncertainty, the dose algorithm could not include a factor relating to operator.

\section{Influence of phone use environment} Urban/rural use

A small $(13 \%-14 \%)$ difference in average power levels was found in the SMP study between subjects using phones mainly 
Figure 2 Surface and axial views of 1 $\mathrm{cm} 3 \mathrm{SAR}(\mathrm{W} / \mathrm{kg})$ distributions in the GRIDMASTER brain at $800-900 \mathrm{MHz}$ and $1800 \mathrm{MHz}$ respectively, for a phone held on the right side (the colour scale used in all views is the same)

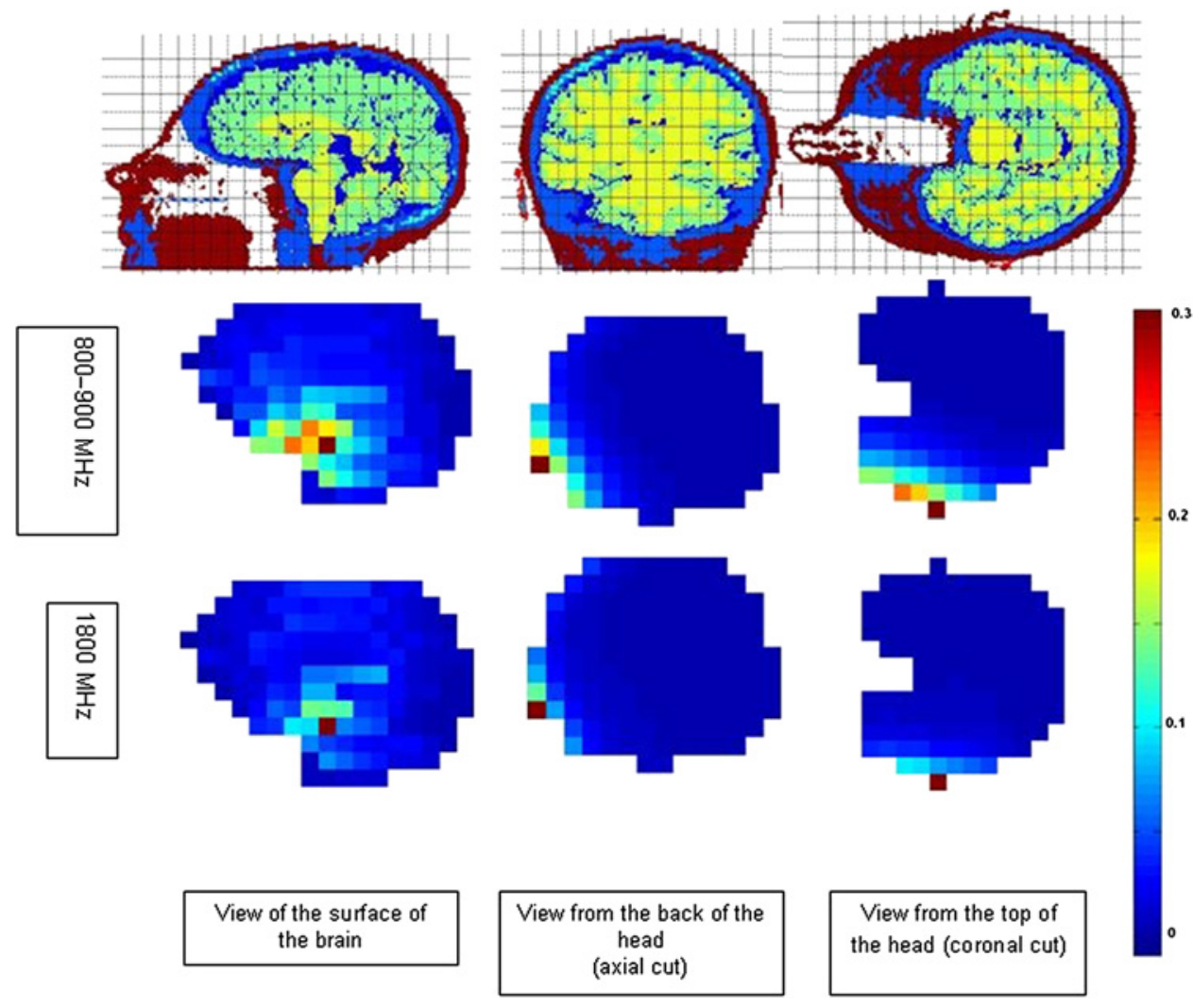

in rural versus urban environments. ${ }^{28}$ This was mainly driven by Sweden, where volunteer selection covered sparsely populated areas. Few rural users were included in SMP studies elsewhere. Base-station data from Sweden ${ }^{36}$ showed greater urban/rural differences (factor of 1.6-1.9 depending on time of day), but data were for 1 week and one operator only.

An urban/rural factor was therefore included in the dose algorithm only where the study covered a large proportion of the country (Israel and New Zealand): based on SMP and basestation studies, average output power was estimated to be $30 \%$ greater in rural users than in urban users for second-generation phones.

\section{Use while moving or stationary}

No difference in average power levels was found in the SMP study between users using phones mainly while moving in a vehicle and those reporting mainly stationary use. ${ }^{28}$ This was initially surprising because networks must hand-over a moving phone from one base-station to another, and with each handover, the power returns to a high level before APC lowers output to optimum level. Handovers, however, occur frequently in stationary situations because networks need to distribute traffic equitably between base-stations, and stationary phone calls may require extra power in built-up areas because of shielding, thus explaining the lack of an effect. The SMP study, moreover, may not have been sufficiently sensitive to detect differences: information available concerned average use (the only information collectable in epidemiological studies) rather than use circumstance of each individual call. Based on the results of the SMP study, it was decided not to differentiate moving and stationary use in the dose algorithm.

\section{Indoor/outdoor}

A difference was found between output power inside and outside buildings in a small study. ${ }^{37}$ A question was added to the
SMP study, but no difference was found. ${ }^{28}$ As no information is available in the Interphone questionnaire on use inside buildings, this variable was not included in the algorithm. This decision is unlikely to bias dose estimates because quality of indoor communications improved over time, leading to gradual increase of indoor use from a low level in the earliest years. However, this is a source of uncertainty.

\section{Influence of DTX}

Use of DTX could not be measured with the SMPs. Data published in $2000^{35}$ suggest that DTX reduced average power by about $30 \%$ once it was enabled in a network. As little information was available from network operators about dates of DTX enablement and because operators which provided information reported it occurred early in GSM networks, it was assumed all GSM networks in the study were DTX enabled from 1994 to 1995

\section{Use of phone-specific SAR values}

The dosimetry database of SARmax for phones in use before and during the Interphone Study included 1233 values. Analysis showed large variation in SAR, with some SARmax measured below $0.01 \mathrm{~W} / \mathrm{kg}$ (current limit of detection) possibly due to measurement errors. Analyses by time period (online appendix figure 3) provided no statistical evidence of a trend over time for a given communication system. The methods and standards used to measure SARmax have evolved over time, so SARmax values are not directly comparable. Analyses (not shown) of SARmax values for more recent models in the database, restricted to measurements in reputable laboratories, showed differences as high as a factor 3 for specific phone models.

For these reasons and because efficiency of phones may partially compensate or exaggerate differences, phone-specific SARmax values were not used in the algorithm: instead, the 
median of available SARmax measurements was assigned to phones in a class. For this, SARmax measured with averaging volumes of 1 or $10 \mathrm{~g}$ were converted to the SAR of the $1 \mathrm{~cm}^{3}$ Gridmaster cell with the highest average SAR for the phone class. Conversion factors were derived from the database of SARmax (1 g) and SARmax (10 g) in the French and Japanese measurements. Single ratios were used for all frequency bands for SARmax (1 g) and for SARmax (10 g) as analyses showed no significant difference across bands (not shown).

\section{Information on phone use from study subjects}

Information on duration and number of calls was reported by Interphone Study subjects by period of use for each phone they had used. Information on laterality of use, call environment and use of hands-free devices was also collected. For time periods for which a subject reported use of hands-free devices, amount of use was reduced by $100 \%, 75 \%, 50 \%$ or $25 \%$, respectively, if devices were used always or almost always, more than half, about half or less than half of the time. If the subject reported a preferred side of use, $90 \%$ of use was assigned to that side of the head and $10 \%$ to the other. Otherwise, $50 \%$ was assigned to each side of the head. Sensitivity analyses were also conducted in which reported laterality was not used at all, and $50 \%$ of use was assigned to each side for all subjects.

\section{Construction of the dose algorithm and application to Interphone Study subjects}

Based on the above, an algorithm was developed for RF dose from mobile phones in terms of cumulative specific energy (CSE) (in joules per kilogram) absorbed at a given location in the brain (l), for a given frequency band $(f)(450,800-900,1500$, $1800-1900 \mathrm{MHz}$ ) and a specific communication system (s) (GSM, CDMA, etc). Location can be anywhere within the Gridmaster cells, and the quantity estimated is the average within the volume of the relevant cells. The algorithm estimates the $\operatorname{CSE}\left(\mathrm{CSE}_{l, f, s}\right)$ absorbed at that location for a given study subject as follows:

$$
\begin{aligned}
\operatorname{CSE}_{l, f, s}= & \sum_{\text {telephone }=1}^{\text {last }}\left[X_{l, c} * \text { Average }_{c}\left(S A R_{x} * \operatorname{Conv}_{1 / 10 g}\right) *\right. \\
& \left.\left(\sum_{\text {operator }=1}^{\text {last }} \sum_{i=1}^{\text {last }} T_{i} * H_{i} * Y_{i, u / r} * P_{i, f, s, o} * O_{i, f, s, o}\right)\right]
\end{aligned}
$$

where:

$i$ denotes month of use within a period defined by operator and phone,

$X_{1, \mathrm{c}}$ proportion of the SARmax received at location $l$, for a phone in class $c$

Average $_{c}$ average over all phones in class $c$ of the argument in parentheses
$\mathrm{SAR}_{\mathrm{x}}$ SARmax (1 or $10 \mathrm{~g}$ ) for phone $x$ in class $c$ from the SARmax measurements of phones.

Conv ${ }_{1 / 10 g}$ conversion factor from SARmax measured for averaging volumes of 1 or $10 \mathrm{~g}$ to SARmax in the Gridmaster cell with highest average SAR.

$T_{\mathrm{i}}$ reported average call time in month $i$ from answers to the questionnaire

$H_{\mathrm{i}}$ modifier for reported use of hands-free devices in month $i$ from the questionnaire (see above)

$\mathrm{Y}_{\mathrm{i}, \mathrm{u} / \mathrm{r}}$ effect of exposure circumstances modifying output power (only urban/rural use-see above)

$P_{\mathrm{i}, \mathrm{f}, \mathrm{s}}$ o proportion of traffic in frequency $f$ and communication system $s$ for operator $o$ at time $i$ based on information from network operators questionnaires. Proportion is 1 if the phone operated in a single band and system. When dual bands/systems were enabled, the relative proportions of traffic were taken to increase linearly from 1.0/0.0 at time of introduction of the second frequency band/system to $\mathrm{p} /(1-\mathrm{p})$-the proportions stated at time of response by the operator-and to remain constant thereafter.

$\mathrm{O}_{\mathrm{i}, \mathrm{f}, \mathrm{s}, \mathrm{o}}$ effect of other modifiers as appropriate (APC and DTX, see above).

The total CSE absorbed at a given location (l) $\mathrm{TCSE}_{l}$ for a particular study subject was obtained by summing over all combinations of communication systems and frequencies he/she used. Risk analyses can be conducted both using $\mathrm{TCSE}_{l}$ as the explanatory variable (assuming that carrier frequencies and their modulation by different communication systems do not modify possible biological effects) and investigating separately the effect of CSE in different frequencies and communication systems. ${ }^{42}$

Based on this algorithm, other metrics can be developed including the time-weighted average of the absorbed power (TWA-SAR), obtained by dividing TCSE by total duration of calls, as well as TCSE and TWA-SAR in different time windows before case diagnosis to explore the hypotheses that risk, if any, may be related to dose rate rather than cumulative dose and that doses in different time windows may have different effects.

\section{Distribution of doses}

Table 1 and figure 3 show the resulting distribution of CSE at tumour location by communication system and frequency among glioma study subjects from the five countries (results for meningioma study subjects are similar-not shown). Substantial differences were seen between technologies, with CSE being highest on average for Advanced Mobile Phone System (AMPS) and lowest for GSM-1800 and CDMA systems.

The distribution of TCSE at the tumour location by cumulative call time, cumulative number of calls and time since start of use of mobile phones is shown in table 2 overall (see online appendix table 2 for specific frequencies and communications systems).

Table 1 Results of dose estimation for glioma study subjects in five Interphone countries: estimated TCSE absorbed at the estimated centre of the

\begin{tabular}{|c|c|c|c|c|c|c|c|}
\hline \multirow{2}{*}{$\begin{array}{l}\text { Communication system and frequency } \\
\text { band }\end{array}$} & \multirow[b]{2}{*}{ Number of subjects } & \multicolumn{6}{|c|}{ TCSE (J/kg) } \\
\hline & & Mean & SD & Min & Max & 25th quartile & 75th quartile \\
\hline AMPS-800 & 669 & 5165 & 24309 & 0 & 515471 & 110 & 2315 \\
\hline DAMPS-800 & 507 & 3946 & 12921 & 0 & 169535 & 53 & 1990 \\
\hline GSM-800/900 & 755 & 2542 & 8858 & 0 & 89907 & 57 & 1279 \\
\hline CDMA-800 & 262 & 164 & 446 & 0 & 4355 & 7 & 92 \\
\hline GSM-1800 & 391 & 467 & 2325 & 0 & 40632 & 3 & 187 \\
\hline CDMA-1900 & 108 & 185 & 622 & 0 & 5479 & 4 & 80 \\
\hline
\end{tabular}
tumour in joules per kilogram, by communication system, up to 1 year before reference date

AMPS, Advanced Mobile Phone System; CDMA, Code Division Multiple Access; DAMPS, Digital Advanced Mobile Phone System; GSM, Global System for Mobile. 


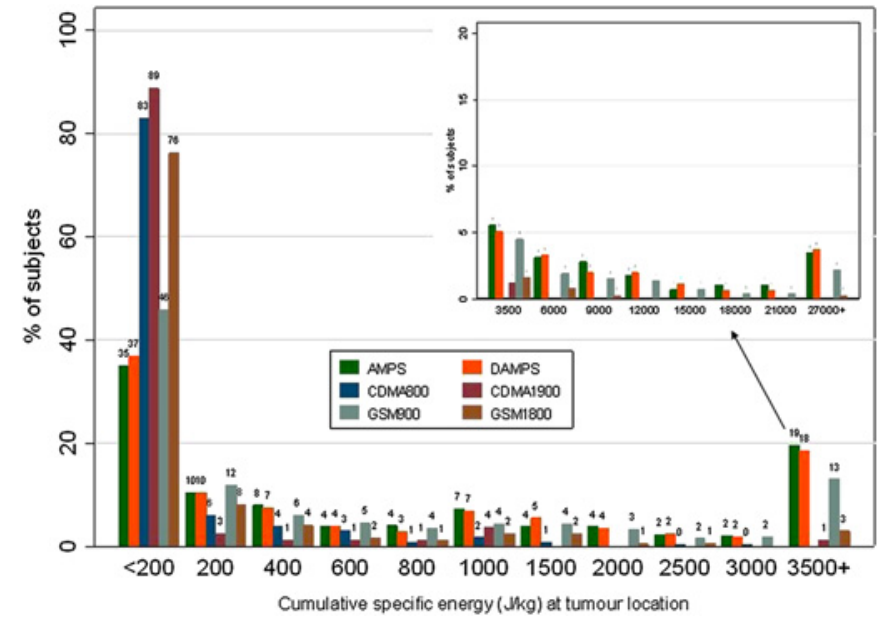

Figure 3 Distribution of estimated CSE (in $\mathrm{J} / \mathrm{kg}$ ) at the origin of the tumour by mobile phone communication system and frequency band. (Glioma study subjects 5 Interphone countries. For controls, location of tumour is taken to be the location of the tumour of the case to which they are matched). AMPS: Advanced Mobile Phone System; D-AMPS: Digital AMPS; CDMA: code division multiple access; GSM: Global System for Mobile. Numbers beside CDMA and GSM indicate the main frequency band (see Appendix 1 for details of the communication systems)

While substantial agreement (34\%) exists between categorisation of subjects by TCSE and cumulative call time ( $\kappa 0.68)$, there is non-negligible misclassification: $37 \%, 21 \%$ and $8 \%$ of subjects, respectively, have 1, 2 or more deciles of difference. Because higher frequencies penetrate less in the brain, the agreement is substantially lower for GSM-1800 (with 5\% having complete agreement and $86 \%$ more than 2 deciles difference) than for lower frequencies (online appendix table 2). Results are similar for cumulative number of calls though $\kappa$ statistics, and agreement are lower. TCSE is also related to time since start of use (table 2 and online appendix table 2), with most subjects with high TCSE being long-term users and most short-term users having low TCSE.

In analysis of variance including the main factors in the dose algorithm, only cumulative call time and tumour location were statistically significant predictors (predicting, respectively, 43\% and $13 \%$ of the variability) for TCSE overall. Results were similar for analogue systems and $800-900 \mathrm{MHz}$ frequency bands. For CDMA 1900, APC was statistically significantly associated with CSE $(p=0.015)$.

\section{DISCUSSION}

While attempts to take into account SAR spatial distribution have been made in epidemiological studies before, ${ }^{20} 43$ this is the first attempt to systematically evaluate and quantify the main parameters thought to influence RF dose from mobile telephone use in an epidemiological study.

This work has necessitated identification of the likely important determinants of SAR, and collection of appropriate information from study subjects, network operators and laboratories involved in SAR measurements to evaluate the importance of each factor. It also necessitated the development of SMPs and the conduct of volunteer studies using these phones.

The main determinants of TCSE from mobile phones were found to be communication system and frequency band, tumour location and amount and duration of mobile phone use. Factors such as APC (except in CDMA networks where its influence is substantial), DTX, urban/rural, moving/stationary and indoor/ outdoor use were found to have a relatively minor influence.

Although TCSE and cumulative call time agree substantially in categorising RF exposures, using only the reported call time creates a complicated mixture of Berkson and classical measurement errors, ${ }^{44}$ particularly at higher frequencies and in CDMA networks. If RF dose is truly associated with brain tumour risk, these exposure assessment errors could bias risk estimates towards the null (no effect) and increase their

Table 2 Distribution of deciles of TCSE absorbed at the location of the tumour (in joules per kilogram) by cumulative call time, cumulative number of calls and time since start of mobile phone use. All frequencies and communication systems. (Glioma study subjects five Interphone countries)

\begin{tabular}{|c|c|c|c|c|c|c|c|c|c|c|c|c|c|c|c|c|c|c|c|c|c|c|c|c|c|c|c|}
\hline \multirow[b]{2}{*}{$\begin{array}{c}\text { Total } \\
\text { cumulative } \\
\text { specific } \\
\text { energy } \\
\text { (deciles) }\end{array}$} & \multicolumn{11}{|c|}{ Cumulative call time (deciles) } & \multicolumn{11}{|c|}{ Cumulative number of calls (deciles) } & \multicolumn{5}{|c|}{ Time since start of use (yrs) } \\
\hline & $\frac{c}{\llcorner}$ & 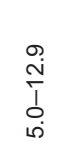 & $\begin{array}{l}0 \\
\dot{p} \\
1 \\
m \\
m\end{array}$ & $\begin{array}{l}0 \\
\dot{0} \\
\frac{1}{m}\end{array}$ & 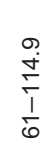 & 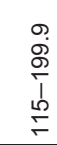 & $\begin{array}{l}\text { Oా } \\
\text { م) } \\
\text { I } \\
\stackrel{0}{0}\end{array}$ & 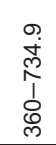 & 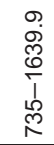 & $\stackrel{+}{+}$ & 唁 & $\begin{array}{l}\frac{0}{\bar{N}} \\
\stackrel{0}{0} \\
\dot{\omega} \\
\bar{v}\end{array}$ & $\begin{array}{l}\stackrel{\nabla}{\infty} \\
\stackrel{b}{b} \\
\stackrel{\sim}{r}\end{array}$ & 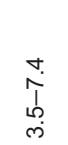 & $\begin{array}{l}\stackrel{\infty}{m} \\
\stackrel{m}{!} \\
\stackrel{\omega}{N}\end{array}$ & 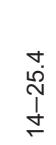 & 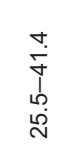 & 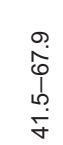 & 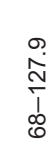 & $\begin{array}{l}\stackrel{\circ}{0} \\
\stackrel{0}{0} \\
i \\
\stackrel{\infty}{\sim}\end{array}$ & $\stackrel{+}{\stackrel{1}{N}}$ & त्ञ & 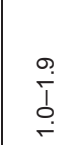 & $\stackrel{\substack{1 \\
1}}{1}$ & $\begin{array}{l}0 \\
1 \\
0 \\
0\end{array}$ & $\stackrel{+}{\circ}$ & 要 \\
\hline$<16.9 \mathrm{~J} / \mathrm{kg}$ & 92 & 44 & 9 & 1 & 2 & 2 & 0 & 0 & 0 & 0 & 150 & 78 & 40 & 22 & 6 & 2 & 1 & 1 & 0 & 0 & 0 & 150 & 70 & 64 & 11 & 5 & 150 \\
\hline $16.9-57.0$ & 10 & 50 & 52 & 29 & 7 & 5 & 0 & 0 & 0 & 0 & 153 & 14 & 31 & 45 & 35 & 16 & 7 & 4 & 1 & 0 & 0 & 153 & 32 & 90 & 29 & 2 & 153 \\
\hline $57.1-135.9$ & 5 & 16 & 37 & 43 & 39 & 14 & 3 & 2 & 0 & 0 & 159 & 8 & 12 & 38 & 26 & 36 & 20 & 14 & 3 & 2 & 0 & 159 & 20 & 93 & 43 & 3 & 159 \\
\hline $136-238.8$ & 0 & 7 & 29 & 28 & 24 & 33 & 8 & 3 & 1 & 0 & 133 & 4 & 10 & 28 & 19 & 21 & 19 & 19 & 11 & 2 & 0 & 133 & 11 & 67 & 51 & 4 & 133 \\
\hline $238.9-449.4$ & 0 & 0 & 17 & 26 & 34 & 32 & 27 & 10 & 2 & 0 & 148 & 1 & 1 & 15 & 24 & 31 & 32 & 15 & 20 & 6 & 3 & 148 & 10 & 75 & 51 & 12 & 148 \\
\hline $449.5-856.4$ & 0 & 1 & 6 & 9 & 16 & 42 & 22 & 37 & 6 & 1 & 140 & 0 & 2 & 2 & 8 & 30 & 22 & 35 & 21 & 14 & 6 & 140 & 6 & 53 & 74 & 7 & 140 \\
\hline $856.5-1069.3$ & 0 & 0 & 2 & 7 & 19 & 21 & 37 & 36 & 23 & 1 & 146 & 0 & 0 & 3 & 8 & 13 & 28 & 34 & 35 & 13 & 12 & 146 & 0 & 51 & 82 & 13 & 146 \\
\hline $1609.4-2909.3$ & 0 & 0 & 0 & 1 & 15 & 18 & 25 & 36 & 31 & 8 & 134 & 0 & 0 & 0 & 5 & 12 & 18 & 26 & 27 & 25 & 21 & 134 & 1 & 39 & 75 & 19 & 134 \\
\hline $2909.4-8242.7$ & 0 & 0 & 0 & 1 & 1 & 6 & 23 & 43 & 41 & 37 & 152 & 0 & 0 & 3 & 1 & 6 & 11 & 19 & 31 & 39 & 42 & 152 & 0 & 33 & 85 & 34 & 152 \\
\hline $8242.8+$ & 0 & 0 & 0 & 0 & 0 & 1 & 4 & 16 & 43 & 105 & 169 & 0 & 0 & 0 & 0 & 1 & 5 & 9 & 19 & 53 & 82 & 169 & 0 & 23 & 95 & 51 & 169 \\
\hline Total & 107 & 118 & 152 & 145 & 157 & 174 & 149 & 183 & 147 & 152 & 1484 & 105 & 96 & 156 & 132 & 168 & 163 & 176 & 168 & 154 & 166 & 1484 & 150 & 588 & 596 & 150 & 1484 \\
\hline $\begin{array}{l}\text { Weighted } \\
\text { kappa- statistic }\end{array}$ & \multicolumn{11}{|c|}{0.68} & \multicolumn{11}{|c|}{0.59} & \multicolumn{5}{|c|}{$0.37^{*}$} \\
\hline $\begin{array}{l}\% \text { agreement } \\
\% 1 \text { decile diff } \\
\% 2 \text { decile diff } \\
\% \text { greater diff }\end{array}$ & \multicolumn{11}{|c|}{$\begin{array}{l}33.83 \% \\
37.06 \% \\
21.50 \% \\
7.61 \%\end{array}$} & \multicolumn{11}{|c|}{$\begin{array}{l}27.02 \% \\
36.86 \% \\
19.68 \% \\
16.44 \%\end{array}$} & \multicolumn{5}{|c|}{$35.31 \%$} \\
\hline
\end{tabular}


uncertainty, making it more likely that real associations with RF dose would not be detected in case-control and cohort studies. ${ }^{44}{ }^{45}$ Efforts to take into account main determinants of dose are therefore important.

There are a number of uncertainties in our estimation of dose, which will need to be investigated further:

- Uncertainties in spatial SAR distribution for each phone class, ${ }^{27}$ particularly in relation to possible differences within classes. This study found clustering of phones related to external phone characteristics only in specific phone positions. ${ }^{31}$ As this varies during normal use, it cannot be used to define phone classes, but regrouping of phones may cause some misclassification.

- Difficulties in localising the probable centre of the tumour. A protocol for localisation was developed and tested by a panel of neuroradiologists. In most cases, neuroradiologists reported high confidence in their evaluation. In some instances, however, particularly when images were not available for localisation, only tumour location was entered into the Gridmaster. The centre was then estimated with a specially developed computer program to find the tumour's centre of gravity (except for meningioma located at the surface of the meninges, close to the skull, for which the centre was estimated as the geometric centre of gravity of the outermost layer of the tumour). Comparison of centres predicted in this way with tumour centres estimated by neuroradiologist showed good agreement. ${ }^{46}$

- Lack of reliable systematic information on SARmax and phone efficiency for specific phones used by study subjects and lack of information on parameters influencing APC for specific networks in the study. Average information therefore had to be used, adding uncertainty to the dose estimation.

- Potential error in recall of phone use: Interphone validation studies indicated that recall was subject to moderate systematic but substantial random error. ${ }^{47}$ Systematic errors were larger for duration of calls than number of calls, and phone use was underestimated by light users and overestimated by heavy users. There was little evidence overall that error was different between cases and controls overall, though cases appeared to over-report for more distant time periods. ${ }^{48}$

- Potential error in laterality of use. Little direct data exist on laterality recall errors, and impact of potential recall biases on risk estimates has been the topic of debate. ${ }^{21}$ Since laterality is one of the components of our algorithm, risk estimates can be derived with or without it, thereby shedding some light on this important question.

- Pulsed low-frequency magnetic fields from digital phones. DC electricity flowing from the phone's battery with each pulsed data transmission generates magnetic fields ${ }^{49} 50$ (online appendix figure 4). For GSM phones, these $217 \mathrm{~Hz}$ pulses have frequency components in the extremely low-frequency range, where brain cancer risks have been inconsistently associated with occupational exposures. ${ }^{51}$ Interphone investigated the feasibility of estimating exposure to pulsed magnetic field emissions, but this was beyond the study's resources. Pilot measurements made on SMPs (online appendix 2) demonstrated that CSE and cumulative exposures to pulsed magnetic fields have many of the same determinants (online appendix figures 4-5). Therefore, the cumulative magnitude of pulsed magnetic fields from digital mobile phones may be confounded with TCSE in epidemiological studies.
- Lack of knowledge regarding the biologically relevant dose metric. Although correct specification of dose metric can have a major impact on a study's power, ${ }^{52}$ laboratory studies of effects of non-thermal RF exposures provide little guidance on biophysical mechanisms, if any, and relevant dose metric for RF epidemiology. Cumulative dose is a biologically valid dose measure for many carcinogenic agents, such as ionising radiation, and was therefore used as the main metric in our study, though other metrics such as dose rate and cumulative electric field strength can also be derived from our algorithm.

\section{CONCLUSIONS}

While substantial agreement was found between categorisation of subjects by TCSE and cumulative call time, there was nonnegligible misclassification, related to communication system and tumour location, particularly at higher frequency bands. While this is important for Interphone, it will be particularly important in future epidemiological studies in which a greater proportion of subjects will have used higher 1500, 1800-1900, $2200 \mathrm{MHz}$ frequency bands and communication systems with more efficient power control like CDMA and UMTS. Efforts to investigate different dose metrics and to quantify the uncertainties described above in future, more detailed studies are needed to improve dose estimation and reduce uncertainty in case-control and cohort studies of brain tumours and RF exposure from mobile telephones.

Acknowledgements The authors would like to thank the following persons for their contribution to this work: Lesley Richardson, Canada, for her important role in the coordination of the Interphone Study and for insightful comments and suggestions during the development of the algorithm and the preparation of the current manuscript; Lucia Ardoino, Italy, for her work in the early stages of this project testing SMPs and studying output power in different settings; the Interphone Principal Investigators from Australia, Canada, France, Israel and New Zealand for the use of their data for descriptive dose analyses; mobile phone manufacturers (Alcatel, Ericsson, Motorola, Nokia) for the development and loan of the SoftwareModified Phones; Edward Mantiply (US Federal Communications Commission) for suggesting analysis of the correlation between the RF dose and the pulsed magnetic field exposures.

Funding This Interphone Study was conducted with funding from the European Fifth Framework Program, 'Quality of Life and Management of Living Resources' (contract OLK4-CT-1999901563) and the International Union against Cancer (UICC). The UICC received funds for this purpose from the Mobile Manufacturers' Forum and GSM Association. Provision of funds to the Interphone Study investigators via the UICC was governed by agreements that guaranteed Interphone's complete scientific independence. The terms of these agreements are publicly available at http://www. iarc.fr/ENG/Units/RCAd.html/. Specific additional funding for the RF dose algorithm and the analyses presented here were provided by the German Bundesamt fuer Strahlenschutz, the French Fondation Santé et Radiofréquences and the Committee to Promote Research on the Possible Biological Effect of Electromagnetic Fields, Ministry of Internal Affairs and Communications, Japan. The findings and conclusions in this paper have not been formally disseminated by the National Institute for Occupational Safety and Health and should not be construed to represent any agency determination or policy.

Competing interests JB, EC, ID, JF, SM, MM, MT, RV, MV, PV and KW have no conflict of interest to declare. JW works for the research centre of France Telecom, as did NV from 2004 to 2005 and from 2010.

Patient consent Consent was obtained from patient or next of kin of the patient was too ill or had died and the next of kin was interviewed as a proxy.

Ethics approval This study was conducted with the approval of the ethical committee of the International Agency for Research on Cancer (IARC) and appropriate local and national Institutional Review Boards (IRBs) in participating countries.

Contributors All authors participated in the conception and design, analysis and interpretation of data, the drafting of the article or its critical revision for important intellectual content and approved the version to be published.

Provenance and peer review Not commissioned; externally peer reviewed. 


\section{REFERENCES}

1. Cardis E, Richardson L, Deltour I, et al. The INTERPHONE study: design, epidemiological methods, and description of the study population. Eur J Epidemiol 2007;22:647-64.

2. Dreyer NA, Loughlin JE, Rothman KJ. Cause-specific mortality in cellular telephone users. JAMA 1999;282:1814-16.

3. Johansen C, Boice $\mathrm{J} J \mathrm{~J}$, McLaughlin J, et al. Cellular telephones and cancer-a nationwide cohort study in Denmark. J Nat/ Cancer Inst 2001;93:203-7.

4. Schüz J, Jacobsen $\mathrm{R}$, Olsen $\mathbf{J H}$, et al. Cellular telephone use and cancer risk: update of a nationwide Danish cohort. J Natl Cancer Inst 2006;98:1707-13.

5. Auvinen A, Hietanen M, Luukkonen R, et al. Brain tumors and salivary gland cancers among cellular telephone users. Epidemiology 2002;13:356-9

6. Christensen HC, Schüz J, Kosteljanetz M, et al. Cellular telephones and risk for brain tumors: a population-based, incident case-control study. Neurology 2005;64:1189-95.

7. Hardell L, Näsman A, Påhlson A, et al. Use of cellular telephones and the risk for brain tumours: A case-control study. Int J Oncol 1999:15:113-16.

8. Hardell L, Carlberg M, Hansson MK. Pooled analysis of two case-contro studies on the use of cellular and cordless telephones and the risk of benign brain tumours diagnosed during 1997-2003. Int J Oncol 2006;28:509-18.

9. Hardell L, Carlberg M, Hansson Mild K. Pooled analysis of two case-control studies on use of cellular and cordless telephones and the risk for malignant brain tumours diagnosed in 1997-2003. Int Arch Occup Environ Health 2006; 79:630-9

10. Hardell L, Mild KH, Carlberg M, et al. Tumour risk associated with use of cellular telephones or cordless desktop telephones. World J Surg Oncol 2006;4:74

11. Hepworth SJ, Schoemaker MJ, Muir KR, et al. Mobile phone use and risk of glioma in adults: case-control study. BMJ 2006;332:883-7.

12. Hours M, Bernard M, Montestrucq L, et al. [Cell Phones and Risk of brain and acoustic nerve tumours: the French INTERPHONE case-control study.] (in French). Rev Epidemiol Santé Publique 2007:55:321-32.

13. Inskip PD, Tarone RE, Hatch EE, et al. Cellular-telephone use and brain tumors. N Engl J Med 2001;344:79-86.

14. Lahkola A, Auvinen A, Raitanen J, et al. Mobile phone use and risk of glioma in 5 North European countries. Int J Cancer 2007:120:1769-75.

15. Lahkola A, Salminen T, Raitanen J, et al. Meningioma and mobile phone use-a collaborative case-control study in five North European countries. Int J Epidemiol 2008:37:1304-13.

16. Lönn S, Ahlbom A, Hall P, et al. Long-term mobile phone use and brain tumor risk Am J Epidemiol 2005:161:526-35.

17. Muscat JE, Malkin MG, Thompson S, et al. Handheld cellular telephone use and risk of brain cancer. JAMA 2000;284:3001-7.

18. Schüz J, Böhler E, Berg G, et al. Cellular phones, cordless phones, and the risks of glioma and meningioma (Interphone Study Group, Germany). Am J Epidemiol 2006;163:512-20.

19. Klaeboe L, Blaasaas KG, Tynes T. Use of mobile phones in Norway and risk of intracranial tumours. Eur J Cancer Prev 2007;16:158-64.

20. Takebayashi T, Varsier N, Kikuchi Y, et al. Mobile phone use, exposure to radiofrequency electromagnetic field, and brain tumour: a case-control study. Br J Cancer 2008;98:652-9.

21. Interphone Study Group. Brain tumour risk in relation to mobile telephone use: results of the INTERPHONE international case-control study. Int J Epidemiol 2010;39:675-94

22. IEC. Procedure to Measure the Specific Absorption Rate (SAR) for Hand-Held Mobile Wireless Devices in the Frequency Range of $300 \mathrm{MHz}$ to $3 \mathrm{GHz}^{\prime \prime} 2006$. IEC 62209-1 Standard. International Electrotechnical Commission (IEC), Geneva. 2005.

23. IEEE. Recommended Practice for Determining the Peak Spatial-Average Specific Absorption Rate (SAR) in the Human Head from Wireless Communications Devices: Measurement Techniques. EEE 1528. New York: The Institute of Electrical and Electronics Engineers, Inc, 2003

24. Kainz W, Christ A, Kellom T, et al. Dosimetric comparison of the specific anthropomorphic mannequin (SAM) to 14 anatomical head models using a novel definition for the mobile phone positioning. Phys Med Biol 2005:50:3423-45

25. Wiart J, Mittra R, Chaillou S, et al. The analysis of human head interaction with a hand-held mobile using the non-uniform FDTD. In Proceedings IEEE AP-S Wireless Commun. Conf Nov. 1998, pp.77-80.
26. GridMaster Computer Program. Düsseldorf: Vompras, 2007.

27. Cardis E, Deltour I, Mann S, et al. Distribution of RF energy emitted by mobile phones in anatomical structures of the brain. Phys Med Biol 2008;53:2771-83.

28. Vrijheid M, Mann S, Vecchia P, et al. Determinants of mobile phone output power in a multinational study: implications for exposure assessment. Occup Environ Med 2009:66:664-71

29. Cenelec. European Standard "Basic Standard for the Measurement of Specific Absorption Rate Related to Human Exposure to Electromagnetic Fields from Mobile Phones (300 MHz-3 GHz). CENELEC EN50361. Brussels: European Committee for Electrotechnical Standardization (CENELEC), 2001

30. CENELEC. Considerations for Human Exposure to Electromagnetic Fields from Mobile Telecommunication Equipment (MTE) in the Frequency Range $30 \mathrm{MHz}-6 \mathrm{GHz}$. CENELEC prES 59005. Brussels: European Committee for Electrotechnical Standardization, 1997. (CENELEC).

31. Deltour I, Wiart J, Taki M, et al. Classification of 3-dimensional SAR distributions emitted by mobile phones. Bioelectromagnetics. Published Online First: 21 Jun 2011 doi:10.1002/bem.20684

32. Wake K, Varsier N, Watanabe S, et al. The estimation of 3D SAR distributions in the human head from mobile phone compliance testing data for epidemiological studies. Phys Med Biol 2009:54:5695-706.

33. Cohen J. A coefficient of agreement for nominal scales. Educ Psychol Meas 1960;20:37-46.

34. Jackson JD. Classical Electrodynamics. 3rd edn. New York: John Wiley and Sons, 1999.

35. Wiart J, Dale C, Bosisio AD, et al. Analysis of the influence of the power control and discontinuation transmission on RF exposure with GSM mobile phones. IEEE Transactions on Electomagnetic Compatibility 2000;42:376-85

36. Lönn S, Forssen U, Vecchia $P$, et al. Output power levels from mobile phones in different geographical areas; implications for exposure assessment. Occup Environ Med 2004;61:769-72.

37. Ardoino L, Barbieri E, Vecchia P. Determinants of exposure to electromagnetic fields from mobile phones. Radiat Prot Dosimetry 2004;111:403-6.

38. Berg G, Schüz J, Samkange-Zeeb F, et al. Assessment of radiofrequency exposure from cellular telephone daily use in an epidemiological study: German Validation study of the international case-control study of cancers of the brain-INTERPHONE-Study. $J$ Expo Anal Environ Epidemiol 2005:15:217-24.

39. Hillert L, Ahlbom A, Neasham D, et al. Call-related factors influencing output power from mobile phones. J Expo Sci Environ Epidemiol 2006;16:507-14.

40. Erdreich LS, Van Kerkhove MD, Scrafford CG, et al. Factors that influence the radiofrequency power output of GSM mobile phones. Radiat Res 2007;168:253-61.

41. Kelsh MA, Shum M, Sheppard AR, et al. Measured radiofrequency exposure during various mobile-phone use scenarios. J Expo Sci Environ Epidemiol 2010:1-12.

42. Cardis E, Armstrong BK, Bowman JD, et al. Risk of brain tumours in relation to estimated RF dose from mobile phones - results from five Interphone countries. Occup Environ Med 2011. doi:10.1136/oemed-2011-100155.

43. Larjavaara S, Mäntylä R, Salminen T, et al. Incidence of gliomas by anatomic location. Neuro Oncol 2007:9:319-25.

44. Armstrong BK, White E, Saracci R. Principles of Exposure Measurement in Epidemiology. Oxford: Oxford University Press, 1992.

45. Armstrong BG. The effects of measurement errors on relative risk regressions. Am J Epidemiol 1990;132:1176-84.

46. Vrijheid M, Cardis E, Varsier N, et al. Tumour localisation in the Interphone international study of mobile phone radiofrequency exposure and brain tumour risk. (in preparation)

47. Vrijheid M, Cardis E, Armstrong BK, et al. Validation of short-term recall of mobile phone use for the Interphone study. Occup Environ Med 2006:63:237-43.

48. Vrijheid M, Armstrong BK, Bédard D, et al. Recall bias in the assessment of exposure to mobile phones. J Expo Sci Environ Epidemiol 2009;19:369-81.

49. Linde T, Mild KH. Measurement of low frequency magnetic fields from digital cellula telephones. Bioelectromagnetics 1997;18:184-6

50. Tuor M, Ebert S, Schuderer J, et al. Assessment of ELF exposure from GSM handsets and development of an optimized RF/ELF exposure setup for studies of human volunteers. Foundation for Research on Information Technologies in Society, Zurich 2005. BAG Reg. No. 2.23.02.-18/02.001778.

51. WHO (World Health Organization). Extremely Low Frequency Fields. Environmental Health Criteria Monograph No.238. Vol 238. Geneva, Switzerland: WHO, 2007.

52. Caroll RJ, Ruppert D, Stefanski LA, et al. Measurement Error in Nonlinear Models A Modern Perspective 2nd edn. Boca Raton Fl: CRC Press, 2006. 